\title{
Rückmeldungen zur ersten Ausgabe im neuen Verlag
}

\author{
Mark Vetter ${ }^{1}$
}

Published online: 20 June 2019

(c) The Author(s) 2019

Liebe Leserinnen und Leser,

nun halten Sie im 69. Jahrgang der KN bereits die zweite Ausgabe im neuen Verlag in den Händen. Oder Sie lesen diese Ausgabe online. Leider hat die erste Ausgabe im Jahr 2019 auf sich warten lassen. Der Grund dafür bestand darin, dass sich im neuen Verlag noch nicht alle Prozesse so eingespielt haben, wie dies bei unserem früheren Verlag der Fall war. Außerdem ist der Workflow im Springer-Verlag ein anderer. Ich möchte mich auf diesem Wege für das verspätete Erscheinen entschuldigen. Wir haben diesmal alles darangesetzt, rechtzeitig zu erscheinen.

Naturgemäß ist in einem anderen Verlag das Erscheinungsbild unserer Zeitschrift ein anderes. Jeder Verlag hat seine Standards oder Vorgaben und damit auch einen bestimmten Stil, der der Wiedererkennung der Druckerzeugnisse dienen soll. Wir kennen dies aus der Redaktion größerer Kartenwerke wie beispielsweise Atlanten. Die einzelnen Karten in so einem Gesamtwerk unter einem Deckel sollen natürlich auch in einem ähnlichen Duktus erscheinen.

Eines ist jedoch gleich geblieben - unabhängig von Layout oder Format: die Zielstellung der Zeitschrift. Es geht nach wie vor um die Informationsweitergabe. Dies gilt sowohl für den wissenschaftlichen Teil als auch für den Nachrichten- bzw. Informationsteil.

Im Hinblick auf den Nachrichtenteil arbeiten wir immer noch an einer Optimierung. Daher gibt es auch in dieser Ausgabe noch Verbesserungspotenzial. Aller Voraussicht nach können wir den Informationsteil eigenständig erstellen, d.h., unabhängig von den Lay-out-Vorlagen des Verlages.

Die Anforderungen an den wissenschaftlichen Teil haben sich besonders deutlich verändert. Diesen Erfordernissen wollen wir mit dem Verlagswechsel Rechnung tragen. Wissenschaftlerinnen und Wissenschaftlern müssen heute nach den Prinzipien der digitalen Nachhaltigkeit arbeiten. Dies

\section{Mark Vetter}

mark.vetter@fhws.de

1 Würzburg, Germany gilt für die Erstellung neuer Texte wie auch für die Rezeption der Texte von Kolleginnen oder Kollegen. Literaturangaben, Zitationen, Abbildungen etc. müssen digital verfügbar und damit digital wiederverwendbar sein. Anders als in der Vergangenheit ist dies der große Mehrwert der gegenwärtigen Veröffentlichungskultur im Springer-Verlag. Dies ist die hybride Verfügbarkeit der Beiträge, digital wie analog. Dank der Möglichkeit der Online-First-Veröffentlichung, d.h. der Herstellung der schnellen Öffentlichkeit von wissenschaftlichen Erkenntnissen in einer international anerkannten Sprache, ist die Publikation von Artikeln in unserer Zeitschrift viel attraktiver geworden. Dies zeigt sich gegenwärtig auch in einer gestiegenen Zahl an Zusendungen von Manuskripten aus der ganzen Welt.

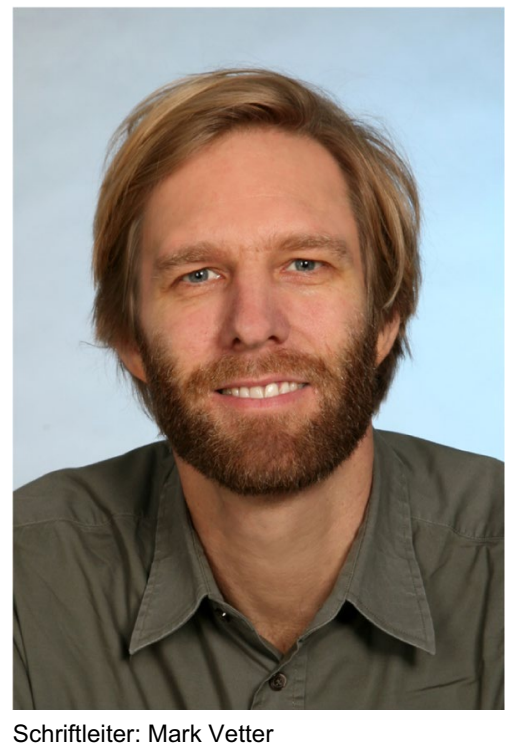

Somit steht die Onlineveröffentlichung im Vordergrund und wir sind sehr froh, dass wir über link.springer.com einen digitalen Auftritt mit hoher wissenschaftlicher Reputation haben. Aber der DGfK ist auch die Printfassung unserer Zeitschrift wichtig. Daher freuen wir uns, dass die KN auch gedruckt in guter Qualität erscheint. Durch die oben 
genannten Rahmenbedingungen, die aus der Onlinewelt kommen, entsteht ggf. der Eindruck, dass der wissenschaftliche Teil unserer Zeitschrift eine Aneinanderreihung einzelner Artikel ist, weil wir zwischen den Artikeln ,WeißRaum“ finden. Das ist so, aber dies lässt sich in den neuen Produktionsprozessen nicht vermeiden. Aber vielleicht ist dies gar nicht so dramatisch, es gibt sogar Leserinnen und Leser, die diesen Umstand als angenehm und wünschenswert empfinden. Im Hinblick auf den Nachrichtenteil werden wir weiter mit Nachdruck an einer inhaltlichen und vom äußeren Erscheinungsbild her individuellen und interessanten Gestaltung arbeiten. Nur: Dafür benötigen wir unbedingt Ihre Mithilfe. Bitte versorgen Sie uns mit Nachrichten und Informationen für den Vereinsteil. Wir freuen uns darauf.
Herzlichst,

\section{Mor unter}

Open Access This article is distributed under the terms of the Creative Commons Attribution 4.0 International License (http://creativeco mmons.org/licenses/by/4.0/), which permits unrestricted use, distribution, and reproduction in any medium, provided you give appropriate credit to the original author(s) and the source, provide a link to the Creative Commons license, and indicate if changes were made.

Viel Freude beim Lesen, 